\title{
Phenotypic characteristics of three indigenous chicken genotypes in Bangladesh
}

\author{
K.N. Monira ${ }^{1 *}$ and S.S. Hussain ${ }^{2}$ \\ ${ }^{1}$ Bangladesh Livestock Research Institute, Savar, Dhaka- 1341, Bangladesh. ${ }^{2}$ Department of Animal \\ Breeding and Genetics, Bangladesh Agricultural University, Mymensing-2202, Bangladesh
}

\begin{abstract}
Poultry contributes the largest parts of animal-source foods. The International Food Policy Research Institute (IFPRI) has estimated that by year 2015, poultry will account for $40 \%$ of all animal protein. Indigenous chicken have a capacity to resist disease, able to utilize low quality feeds and their products are preferred by consumers. There views were collected to indigenous chicken production scenarios and their characteristics and to synthesize the information for gathering knowledge. The review areas were all over Bangladesh but our studied areas were Baraigram, Natore; Sarail, Brahmonbaria and Bandarban Hill tract and genotypes were Indigenous, Aseel and Hilly chicken. The production or management system was backyard scavenging and semi-scavenging type for Indigenous, Hilly and Aseel female chicken but for Aseel male it is used intensive system for breeding program; which was characterized by small flock size. The average number of chicken per house-hold was almost same in Baigram and Sarail (7.93 \pm 4.46 and 7.69 \pm 2.75 ); but lower in Bandarban Hill district (5.11 \pm 1.78$)$. Indigenous, Aseel and Hilly chickens have a large morphological variation. The percent of plumage color of Indigenous chicken was 35\% black brownish, 30\% brown with black strip, completely black 30\% and golden red 5\%; in Aseel chicken was as $45 \%$ golden red, $30 \%$ black brownish, completely black $25 \%$ and in Hilly chickens have $50 \%$ black brownish, $45 \%$ brown with black strip and 5\% black plumage color. $90 \%$ of indigenous chicken have yellow shank while 10\% black shank. All the Aseel chickens have yellow shank. The shank color of Hilly chicken was as 50\% yellow, 39\% white and 11\% black. The study revealed that indigenous chicken and Hilly chicken laid mainly white colored eggs and Aseel chickens laid mainly light brown egg. Feeds were not usually supplemented in all three genotypes reared under scavenging system. Chickens pick up grains such as rice, vegetables, green grass, insect, earthworm etc. from the yard, as chickens have reared under scavenging system. Different types of housing were used for chickens in the selected sites. Approximately $30 \%, 40 \%$ and $60 \%$ of farmers kept chickens in their living houses in cases of Indigenous chickens, Aseel and Hilly chicken, respectively. No farmer used artificial lighting for the chickens. So, the study was performed to review indigenous chicken production scenarios may serve as an important base of Ph.D research and may help to take proper planned to conserve of these three genotypes.
\end{abstract}

(Key words: Review, Phenotype Characterization, Indigenous, Chicken Genotypes.)

\section{Introduction}

Bangladesh has a rich heritage of indigenous poultry germplasm, which strongly supported decisive measures for conserving indigenous genetic resources. The indigenous chicken may be classified into three major groups:
Aseel breed, Hilly and Indigenous (Deshi) (Okada et al., 1987; Faruque et al., 2010). Aseel is the only breed of chicken in Bangladesh (Faruque et al., 2010, Okada et al., 1988). It is the heaviest chicken among the existing breeds and varieties of indigenous chicken in Bangladesh, the highest weight

*Corresponding author: monirablri@yahoo.com

Bang. J. Livs. Res. Special Vol. 21-25, 2018: P. 10-23, ISSN 1022-3851 
being $6 \mathrm{~kg}$ (Bhuiyan et al., 2005; Yamamoto, 2010). Aseel chicken is predominantly reared in the home stead area of Sarail upazilla of Brahmanbaria district. Aseel has been bred there as a game bird for many centuries, specifically for its aggressive behavior. It is a very powerful bird having large bone, with broad shoulders, an upright stance, heavily muscled hips and square shanks, strong and curved neck and short beak. Indigenous chicken mostly of non-descript in nature. They are widely distributed throughout the country and also called Deshi chickens (Okada et al., 1987). Deshi chickens are more genetically diverse, well adapted and more resistant to diseases. Deshi chickens are easy to establish for low income families. Hilly chicken observed in Chottogram Hill Tract. Hilly chickens are very prominent in muscularity and vigourisity and reared for local consumption and its egg and meat have a unique taste, is regarded as a delicacy also popular among consumers.

The indigenous chicken population of Bangladesh has been undergoing genetic erosion since the 1960s following the introduction of improved stock from developed countries. Efforts to sustain commercial hybrid broiler and layer chicken farming under intensive and semi-intensive production models have been tested but efficiency of systematic characterization screening breed improvement and conservation programs with the indigenous Deshi chickens at the smallholder village levels (in-situ) of Bangladesh are yet to be tested. This in turn may help to sustain village chicken production system in Bangladesh and could be a useful micro-economic strategy in the on-going poverty alleviation process in the country (Bhuiyan et al., 2005). Indigenous chickens constitute nearly $80 \%$ of the total chicken population of the country. About $89 \%$ of the rural livestock-holders rear chickens and the average number per household are 6.8 numbers. The production system for indigenous chickens is small holder backyard scavenging in nature with each family keeping an average of 6-7 chickens to meet family requirements. A cash income is derived from them when necessary. Indigenous chickens produce about $75 \%$ of the eggs and $78 \%$ of the meat consumed domestically (Bhuiyan et al., 2005 and Faruque et al., 2010).

The poultry industry is one of the faster growing and most promising industries in the agricultural sector of Bangladesh. Annual average growth rate in the commercial chicken is satisfactory. On the other hand, the growth rate of indigenous chickens is not satisfactory as evident from the supply of egg and meat in the market. Though poultry industry has evidenced faster growth in the livestock sector, still there is a huge gap between supply and demand of poultry meat and eggs. For example, per head annual consumption of eggs in the country is 95 against the minimum requirement of 104 eggs.

Morphological characteristics and production performance variations of some Bangladeshi chickens have been reported by Islam et al. (2011). Attempt has been taken to make for genetic improvement ex situ in institutional flock under intensive management system but information on the production potential of these flocks in situ i.e. in their home tract is not enough. Maximum survey data were collected by interview method and proper recording was not done in situ. However, for real genetic progress, it is essential to use the actual data. Therefore, the present study was 
performed to review indigenous chicken production scenarios and may help to take proper plan to conserve of these three genotypes in Bangladesh. Reviewed data of morphological and production parameters of Indigenous, Aseel and Hilly chicken are shown in Table 1, 2 and 3 respectively.

Table 1. Traits values of Indigenous chickens at different conditions

\begin{tabular}{|c|c|c|c|}
\hline \multirow{2}{*}{$\begin{array}{l}\text { Rearing } \\
\text { system }\end{array}$} & \multicolumn{2}{|l|}{ Traits } & \multirow[t]{2}{*}{ Source } \\
\hline & Morphological & Production & \\
\hline $\begin{array}{l}\text { Backyard } \\
\text { scavenging } \\
\text { system }\end{array}$ & $\begin{array}{l}\text { Plumage colour- black }(75 \%) \text {, red }(25 \%) \\
\text { Skin colour-white }(82 \%) \text {, yellow }(17 \%) \\
\text { Shank color- black }(39 \%) \text {, yellow } \\
(32 \%) \text {, White }(29 \%) \text {; Egg shell colour- } \\
\text { Light brown }(67 \%) \text { white }(27 \%) \\
\text { Comb type-single }\end{array}$ & $\begin{array}{l}\text { Age of } 1^{\text {st }} \text { egg: } 175 \mathrm{~d} \\
\text { Mature body wt. } 1-1.3 \mathrm{~kg} \\
\text { Egg prod/hen/year- } 45-50 \text { no. }\end{array}$ & Bhuiyan et al., 2005 \\
\hline $\begin{array}{l}\text { Scavenging } \\
\text { and Semi- } \\
\text { intensive } \\
\text { system }\end{array}$ & $\begin{array}{l}\text { Plumage colour- male/female - } \\
\text { colored/white } \\
\text { Skin color-white/yellow } \\
\text { Shank color-Male/female-yellow/white } \\
\text { Comb type-single }\end{array}$ & $\begin{array}{l}\text { Age of } 1^{\text {st }} \text { egg: } 156 \mathrm{~d} \\
\text { Mature body wt. male }-1.8 \\
\mathrm{~kg} \text {, female- } 1.4 \mathrm{~kg} \\
\text { Egg prod/hen/year-36 no. } \\
\text { Egg production } \% 24-36 \mathrm{wk} . \\
50 \text { no.; Average egg wt.- } 43 \mathrm{~g} \\
\text { Cluster per year-3-4 }\end{array}$ & Faruque et al., 2010 \\
\hline $\begin{array}{l}\text { Locations: } \\
10 \text { districts } \\
\text { Semi- } \\
\text { intensive } \\
\text { system }\end{array}$ & $\begin{array}{l}\text { Plumage colour- male/female - } \\
\text { colored/white } \\
\text { Skin colour-white/yellow } \\
\text { Shank color-Male/female-yellow/white } \\
\text { Comb type-single }\end{array}$ & $\begin{array}{l}\text { Gene frequencies of } \mathrm{B}^{\mathrm{A}}, \mathrm{B}^{\mathrm{C}} \\
\text { and } \mathrm{B}^{7} \text { were seen in low } \\
\text { frequency though the } \mathrm{B}^{\mathrm{M}} \text { was } \\
\text { comparatively high frequency } \\
\text { in all populations. }\end{array}$ & $\begin{array}{l}\text { Yamamoto et al., } \\
2010\end{array}$ \\
\hline $\begin{array}{l}\text { Locations: } 8 \\
\text { districts } \\
\text { Semi- } \\
\text { intensive } \\
\text { system }\end{array}$ & $\begin{array}{l}\text { The comb shape and the colors of } \\
\text { earlobe, feather and shank was studied. } \\
\text { The pea }(\mathrm{P}) \text {, rose }(\mathrm{R}) \text {, and crest }(\mathrm{Cr}) \text { loci } \\
\text { controlling the comb shape, the barring } \\
\text { (B), extension of black (E), inhibitor (I) } \\
\text { and silver (S) loci controlling the } \\
\text { feather color, and the Id locus for shank } \\
\text { color were investigated. }\end{array}$ & $\begin{array}{l}\text { The comb shape (the P, R and } \\
\text { Cr loci), earlobe color, feather } \\
\text { color (the B, E, I and S loci) } \\
\text { and the shank color (the Id } \\
\text { locus). Gene frequencies were } \\
\text { estimated at these } 8 \text { loci and } \\
\text { at four blood group loci (The } \\
\text { Ea-A, Ea-B, Ea-D and Ea-E } \\
\text { loci). }\end{array}$ & Okada et al.,1987 \\
\hline $\begin{array}{l}\text { Semi- } \\
\text { intensive } \\
\text { system }\end{array}$ & $\begin{array}{l}\text { Comb shape }-99 \% \text { single plumage } \\
\text { color- }(24 \%) \text { white with red colored } \\
(1 \%) \text {. shank color- white }(52 \%) \text { black } \\
(36 \%) \text { yellow }(10 \%) \text {. skin color- white } \\
(47 \%) \text {. }\end{array}$ & $\begin{array}{l}\text { The indigenous chicken laid } \\
48.5 \% \text { white colored eggs, } \\
20 \% \text { red brown and } 1 \% \text { red. } \\
\text { The average adult body } \\
\text { weight was measured } \\
961.50 \mathrm{~g} \text {. }\end{array}$ & $\begin{array}{l}\text { Tabassum et } \\
\text { al., } 2012\end{array}$ \\
\hline $\begin{array}{l}\text { Locations: } 2 \\
\text { districts. } \\
\text { Extensive } \\
\text { system. }\end{array}$ & Production- Not mention & $\begin{array}{l}\text { Age at } 1^{\text {st }} \text { laying } 203.4 \mathrm{~d} \\
\text { Days/clutch-18.07 } \\
\text { Egg/clutch- } 15.64 \\
\text { Clutch/year-3.38 } \\
\text { Male mature wt. } 1.28 \mathrm{~kg} \\
\text { Female mature wt.- } 1.08 \mathrm{~kg}\end{array}$ & $\begin{array}{l}\text { Shahjahan et } \\
\text { al., } 2010\end{array}$ \\
\hline
\end{tabular}

d, day; no - Number, WK - weeks, g-gram, Kg- Kilogram 
Locations: 4 plumage pattern- black $-25.49 \%$, districts. grayish- $22.30 \%$ red- $16.40 \%$ the rest Scavenging showed white, multi colored, black with system. white tips, reddish brown and white
Production- Not mention

Islam et al., 2011 with red stripes. Plain heads, yellow shanks and pea comb were observed $51.18 \%, \quad 64.42 \%$ and $50.72 \%$ respectively. Shank feathering-About $97.52 \%$ no feathers on their legs.

Locations: 2 Comb color - bright red (79.4\%), the districts eye color- brown (70.1\%) and red Scavenging (24.3\%). Neck hackle- black (32.7\%) system. and orange $(29 \%$, plumage color- black $(33 \%)$ gold $(31 \%)$ and white $(20 \%)$, earlobe color- red (58\%) white (45.8\%), skin color- white $(98.1 \%)$, shank colorwhitish (56.1\%) and black (30.8\%), comb pattern- mostly single type (86\%).

\begin{tabular}{|c|c|c|}
\hline $\begin{array}{l}\text { Location: } \\
\text { BLRI farm } \\
\text { Intensive } \\
\text { management } \\
\text { system }\end{array}$ & $\begin{array}{l}\text { Plumage color- black brownish } \\
(33.33 \%) \text { followed by white with black } \\
\text { tips }(28.33 \%) \text { and red brownish } \\
(18.33 \%) \text {. Shank color-whitish } 35 \% \\
\text { yellowish } 31.38 \% \text {; black } 11.66 \% \text { and } \\
\text { others } 21.67 \% \text {. egg color- light brown } \\
(62.42 \%) \text { to cream or off white } \\
(30.28 \%)\end{array}$ & $\begin{array}{l}\text { Male: Shank Length }(\mathrm{cm})- \\
\text { 10.35, Wattle length }(\mathrm{cm})- \\
\text { 4.98, Body weight }(\mathrm{Kg})-2.48 \\
\text { Female: Number of eggs/hen } \\
\text { from starting to ten months of } \\
\text { laying - } 108 \text {, egg weight } \\
42.94 \text { fertility }(\%) 89.65, \\
\text { hatchability }(\%), 88.63,\end{array}$ \\
\hline $\begin{array}{l}\text { Locations: } \\
12 \text { districts } \\
\text { Extensive } \\
\text { system }\end{array}$ & $\begin{array}{l}\text { plumage color, black-34.50, red- } 18.17 \text {, } \\
\text { brown- } 36.33 \text {, white- } 11.00 \% \text {, Type of } \\
\text { comb, simple- } 98.67 \text {, rose- } 0.50 \text {, pea- } \\
0.17 \text {, walnut- } 0.67 \% \text {, body feathering, } \\
\text { normal- } 99.83 \text {, frizzled- } 0.17 \% \text {, Color of } \\
\text { beak, white-5.00, blackisk- } 54.17 \text {, } \\
\text { yellow-33.17, bworn- } 7.67 \% \text {, color of } \\
\text { earlobes, red- } 85.83 \text {, white- } 13.33 \text {, } \\
\text { blackisk- } 0.67 \text {, yellow- } 0.17 \text {, color of } \\
\text { skin, white- } 85.83 \text {, pink-5.50, yellow- } \\
5.33 \text {, brown-3.33 }\end{array}$ & Production: not mention \\
\hline $\begin{array}{l}\text { Location: } \\
\text { BLRI farm } \\
\text { Intensive } \\
\text { system }\end{array}$ & Morphology: not mention & $\begin{array}{l}\text { Age at } 1^{\text {st }} \text { egg-160d } \\
\text { Egg production (no.)- } 60.04 \\
(24-40 \text { wks) } \\
\text { Egg weight- } 42.26 \mathrm{~g}\end{array}$ \\
\hline $\begin{array}{l}\text { Semi- } \\
\text { scavenging } \\
\text { system }\end{array}$ & Morphology: not mention & $\begin{array}{l}57.2 \% \text { farm household had-20 } \\
\text { chicken, } 28.8 \% \text { farm } \\
\text { households had } 20-50 \\
\text { chicken and } 2.0 \% \text { household } \\
\text { had } 70-90 \text { chickens. } 79 \% \\
\text { respondents reared native } \\
\text { chicken in combined house } \\
\text { and } 10 \% \text { prepared separate } \\
\text { house }\end{array}$ \\
\hline
\end{tabular}

Production: not mention Biswas et al., 2005




\begin{tabular}{|c|c|c|c|}
\hline $\begin{array}{l}\text { Scavenging } \\
\text { system }\end{array}$ & Morphology: not mention & $\begin{array}{l}\text { A family having } 15 \text { organic } \\
\text { chickens earns Tk. } 1770 / \text { year, } \\
70-80 \% \text { routing management } \\
\text { performed by women. }\end{array}$ & Paul et al., 2003 \\
\hline $\begin{array}{l}\text { Location: } \\
\text { BLRI farm } \\
\text { Intensive } \\
\text { system }\end{array}$ & $\begin{array}{l}\text { Plumage color was reddish black } \\
(33.13 \%) \text {, shank colors- white } \\
(39.87 \%) \text {, yellow }(37.22 \%) \text {, black } \\
(20.04 \%) \text { and mixed }(2.87 \%) \text {, earlobes - } \\
\text { reddish white }(44.79 \%) \text { white }(29.24 \%) \\
\text { and red }(25.97 \%) \text {, skin color - white } \\
(92.22 \%) \text { single comb }(96.12 \%)\end{array}$ & $\begin{array}{l}\text { Plumage color- BW } \\
\text { Red Black - } 1459.60 \mathrm{~g}, \\
\text { Black Red }-1388.32 \mathrm{~g}, \\
\text { Red }-1211.60 \mathrm{~g}, \\
\text { Black }-1285.37 \mathrm{~g} \\
\text { White Black }-1409.72 \mathrm{~g} \\
\text { Red White }-1315.33 \mathrm{~g} \\
\text { and Black }-1208.54 \mathrm{~g}\end{array}$ & Sarker et al., 2014 \\
\hline $\begin{array}{l}\text { Location: Ra } \\
\text { zshahi Distri } \\
\text { ct Extensive } \\
\text { system }\end{array}$ & Morphology- not mention & $\begin{array}{l}\text { Age at } 1^{\text {st }} \text { lay- } 20 \mathrm{wk} \\
\text { Marketable wt.- } 750 \mathrm{~g} \\
\text { Av. Egg production- } 2.92 \\
\text { month }\end{array}$ & Dutta et al., 2013 \\
\hline $\begin{array}{l}\text { Location:No } \\
\text { akhali } \\
\text { district } \\
\text { Extensive } \\
\text { system }\end{array}$ & Morphology: not mention & $\begin{array}{l}\text { Final body wt-366g } \\
\text { Body wt. gain-301 } \\
\text { FCR-3.05 } \\
\text { Mortality-3.77 }\end{array}$ & Sarker et al., 2013 \\
\hline $\begin{array}{l}\text { Location: } \\
\text { Noakhali } \\
\text { district } \\
\text { scavenging } \\
\text { system }\end{array}$ & Morphology: not mention & $\begin{array}{l}\text { Clutch/hen/year-4 } \\
\text { Egg/clutch-17 } \\
\text { Clutch length-19 } \\
\text { Egg prod./hen/year-68 } \\
\text { Value of eggs \& chicken } \\
\text { consumed/month (BTD)-363 } \\
\text { Income both in cash \& } \\
\text { consumption - 433 Tk. }\end{array}$ & Sarkeret al., 207 \\
\hline $\begin{array}{l}\text { Locations: } 2 \\
\text { districts } \\
\text { scavenging } \\
\text { system }\end{array}$ & Morphology: not mention & $\begin{array}{l}\text { Their livelihoods providing } \\
\text { The maximum number of } \\
\text { chicken egg production per } \\
\text { year was } 1026 \text { and the highest } \\
\text { sale from chicken eggs was } \\
\text { taka } 3591 \text {, so poultry have the } \\
\text { potential to assist resource } \\
\text { poor farmers to improve both } \\
\text { social and economic benefits }\end{array}$ & Hussain et al., 2007 \\
\hline $\begin{array}{l}\text { Location: } \\
\text { Jessore } \\
\text { district } \\
\text { scavenging } \\
\text { system }\end{array}$ & Morphology: not mention & $\begin{array}{l}\text { Egg production (no. bird)-44 } \\
\text { Egg weight- } 36.27 \mathrm{~g} \\
\text { Egg production } \%-11.22 \\
\text { Live weight (kg bird)-1.3 } \\
\text { Age at } 1^{\text {st }} \text { eggs (days)-175 } \\
\text { Mortality } \%-14.5\end{array}$ & Ershad, 2005 \\
\hline $\begin{array}{l}\text { Location: } 2 \\
\text { districts } \\
\text { Semi- } \\
\text { scavenging } \\
\text { system }\end{array}$ & Morphology: not mention & $\begin{array}{l}\text { Live wt.-female- } 966.1 \text {, male - } \\
1010 \text {, Clutch size }(\mathrm{d})-15.18 \\
\text { Egg production }(\mathrm{e} / \mathrm{h} / \mathrm{y})-70.65 \\
\text { Egg wt.(g/egg)-44.17 } \\
\text { Hatchability \% at set egg- } \\
87.40\end{array}$ & Ahmed et al., 2012 \\
\hline
\end{tabular}

e/h/y - Egg/hen/year 


\begin{tabular}{|c|c|c|c|}
\hline $\begin{array}{l}\text { Location: } \\
\text { Gaibandha } \\
\text { district, } \\
\text { scavenging } \\
\text { system }\end{array}$ & Morphology: not mention & $\begin{array}{l}\text { Average populations of } \\
\text { chickens were } 8.4,64 \% \text { of the } \\
\text { farmers were low producer } \\
\text { and only } 6 \% \text { higher producers. } \\
\text { In case of consumption, } 74 \% \\
\text { consumed by low producers }\end{array}$ & Billah et al., 2013 \\
\hline $\begin{array}{l}\text { Location: } \\
\text { Aftab Bahu } \\
\text { mukhi Farm } \\
\text { ltd. Intensive } \\
\text { system }\end{array}$ & Morphology: not mention & $\begin{array}{l}\text { Quality chicks, feed, technical } \\
\text { support, vaccines, medicines } \\
\text { and health monitoring are } \\
\text { provided by the ABFL, their } \\
\text { net profit per month } \\
\text { Tk.2000-4000. }\end{array}$ & Aziz et al., 2003 \\
\hline
\end{tabular}

Table 2. Traits values of Aseel chickens at different conditions

\begin{tabular}{|c|c|c|c|}
\hline \multirow{2}{*}{$\begin{array}{l}\text { Rearing } \\
\text { system }\end{array}$} & \multicolumn{2}{|l|}{ Traits } & \multirow[t]{2}{*}{ Source } \\
\hline & Morphological & Production & \\
\hline $\begin{array}{l}\text { Location: } \\
\text { Brahmanbar- } \\
\text { ia district } \\
\text { backyard } \\
\text { scavenging } \\
\text { system }\end{array}$ & $\begin{array}{l}\text { Plumage colour- Deep purple } \\
\text { Skin colour-white/yellow } \\
\text { Shanks- Featherless } \\
\text { Egg shell colour- Brownish }\end{array}$ & $\begin{array}{l}\text { Age of } 1^{\text {st }} \text { egg: } 240-300 \mathrm{~d} \\
\text { Mature body wt.1.07-4.50kg } \\
\text { Egg prod/hen/year-33 no. }\end{array}$ & Bhuiyan et al., 2005 \\
\hline $\begin{array}{l}\text { Location: } \\
\text { Brahmanbar } \\
\text { ia district } \\
\text { Scavenging } \\
\text { and Semi - } \\
\text { intensive } \\
\text { system }\end{array}$ & $\begin{array}{l}\text { Plumage colour- male/female } \\
\text { Silver/Gold } \\
\text { Skin colour-white/yellow } \\
\text { Shank color-Male/female-yellow/white } \\
\text { Egg shell colour- Brownish }\end{array}$ & $\begin{array}{l}\text { Age of } 1^{\text {st }} \text { egg: not mention } \\
\text { Mature body wt. (male) }-4.5 \\
\mathrm{~kg} \text {, female- } 3.5 \mathrm{~kg} \\
\text { Egg prod/hen/year- } 15 \text { no. } \\
\text { Average egg wt.- not mention }\end{array}$ & Faruque et al., 2010 \\
\hline $\begin{array}{l}\text { Locations: } \\
10 \text { districts } \\
\text { Semi- } \\
\text { intensive } \\
\text { system }\end{array}$ & $\begin{array}{l}\text { Plumage colour- male/female } \\
\text { Silver/Gold } \\
\text { Skin colour-white/yellow } \\
\text { Shank color-Male/female-yellow/white } \\
\text { Comb type- pea/rose }\end{array}$ & $\begin{array}{l}\text { Production parameter-not } \\
\text { mention } \\
\text { Worked on - gene frequencies } \\
\text { of } \mathrm{B}^{\mathrm{A}}, \mathrm{B}^{\mathrm{C}} \text { and } \mathrm{B}^{7} \text { were seen in } \\
\text { low frequency though the } \mathrm{B}^{\mathrm{M}} \\
\text { was comparatively high } \\
\text { frequency in all populations. }\end{array}$ & Yamamoto et al., 2010 \\
\hline $\begin{array}{l}\text { Locations: } 8 \\
\text { districts } \\
\text { Semi- } \\
\text { intensive } \\
\text { system }\end{array}$ & $\begin{array}{l}\text { The comb shape and the colors of } \\
\text { earlobe, feather and shank. The pea } \\
\text { (P), rose (R), and crest (Cr) loci } \\
\text { controlling the comb shape, the barring } \\
\text { (B), extension of black (E), inhibitor } \\
\text { (I) and silver (S) loci controlling the } \\
\text { feather color, and the Id locus for } \\
\text { shank color were investigated }\end{array}$ & $\begin{array}{l}\text { Production parameter-not } \\
\text { mention } \\
\text { The comb shape (the P, R and } \\
\text { Cr loci), earlobe color, feather } \\
\text { color (the B, E, I and S loci) } \\
\text { and the shank color (the Id } \\
\text { locus). Gene frequencies were } \\
\text { estimated at these } 8 \text { loci and } \\
\text { at four blood group loci (The } \\
\text { Ea-A, Ea-B, Ea-D and Ea-E } \\
\text { loci). }\end{array}$ & Okada et al., 1987 \\
\hline
\end{tabular}


Locations: 2 Feathercolor: neck/hackles-red in both districts males $(56.14 \%)$ and females $(54.06 \%)$. Semi- Sickle feather color- black in both intensive males $(71.93 \%)$ and females $(54.17 \%)$. saddle feather color-red $(40.35 \%)$ in male and pale brown (58.33\%) in female. Breast feather color- black $(64.91 \%)$ in male and pale brown $(50 \%)$ in female. primary feather color- black in both male and female system

Shank length- male $12.79 \pm$

Sarker et al., 2011

0.13 female- $10.21 \pm 0.25 \mathrm{~cm}$

Ulna/Radius length, male-

$11.04 \pm 0.07 \mathrm{~cm}$ female- 8.96 $\pm 0.23 \mathrm{~cm}$, Keel bone lengthmale, $14.39 \pm 0.19 \mathrm{~cm}$ female $-10.79 \pm 0.23 \mathrm{~cm}$. Adult live weight male- $3749.12 \pm$ $83.44 \mathrm{~g}$ female $-2062.50 \pm$ 105.26 g. Egg prod. 24-48 numbers of clutch/hen/year.

Semi -

intensive

sys.
Body wt.

Male-3.32 kg (1-2yr)

Female-2.43 kg (1-2yr.)

Total egg:21 (min) $74(\max )$

Plumage color in wing region: cock reddish, black, red and blackish.

Hen-red, brown, black, white, blackish, gray

Plumage color in breast region: cock- Egg wt. 38g (min), 56g (max) red, black, blackish, white, gray Hen-reddish, black, blackish

Table 3. Traits values of Hillychickens at different conditions

\begin{tabular}{|c|c|c|c|}
\hline \multirow{2}{*}{$\begin{array}{l}\text { Rearing } \\
\text { system }\end{array}$} & \multicolumn{2}{|l|}{ Traits } & \multirow[t]{2}{*}{ Source } \\
\hline & Morphological & Production & \\
\hline $\begin{array}{l}\text { Location: } \\
\text { Chittagong } \\
\text { district } \\
\text { backyard } \\
\text { scavenging } \\
\text { system }\end{array}$ & $\begin{array}{l}\text { Plumage colour- grey or reddish } \\
\text { Skin colour-yellow } \\
\text { Shanks- Featherless } \\
\text { Egg shell colour- Brownish }\end{array}$ & Production: not mention & Bhuiyan et al., 2005 \\
\hline $\begin{array}{l}\text { Location: } \\
\text { Chittagong } \\
\text { district } \\
\text { Scavenging } \\
\text { and Semi- } \\
\text { intensive } \\
\text { system }\end{array}$ & $\begin{array}{l}\text { Plumage colour- male/female - } \\
\text { white/black } \\
\text { Skin colour-white/yellow } \\
\text { Shank color-Male/female-black/yellow } \\
\text { Comb type-single }\end{array}$ & $\begin{array}{l}\text { Age of } 1^{\text {st }} \text { egg: } 154 \mathrm{~d} \\
\text { Mature body wt. male }-3.5 \\
\mathrm{~kg} \text {, female- } 2.2 \mathrm{~kg} \\
\text { Egg prod/hen } / \text { year- } 32 \mathrm{no} . \\
\text { Egg production } \% 24-36 \mathrm{wk} . \\
49 \text { no.Average egg wt.- } 42 \mathrm{~g}\end{array}$ & Faruque et al., 2010 \\
\hline $\begin{array}{l}\text { Locations: } 10 \\
\text { districts } \\
\text { Semi- }\end{array}$ & $\begin{array}{l}\text { Plumage colour- male/female - } \\
\text { colored/white } \\
\text { Skin colour-white/yellow }\end{array}$ & $\begin{array}{l}\text { Production parameter-not } \\
\text { mention } \\
\text { Worked on - gene frequencies }\end{array}$ & Yamomoto et al., 2010 \\
\hline $\begin{array}{l}\text { intensive } \\
\text { management } \\
\text { system }\end{array}$ & $\begin{array}{l}\text { Shank color-Male/female-yellow/white } \\
\text { Comb type-single }\end{array}$ & $\begin{array}{l}\text { of } \mathrm{B}^{\mathrm{A}}, \mathrm{B}^{\mathrm{C}} \text { and } \mathrm{B}^{7} \text { were seen in } \\
\text { low frequency though the } \mathrm{B}^{\mathrm{M}} \\
\text { was comparatively high } \\
\text { frequency in all populations. }\end{array}$ & \\
\hline $\begin{array}{l}\text { Locations: } 8 \\
\text { districts } \\
\text { Semi- } \\
\text { intensive } \\
\text { system }\end{array}$ & $\begin{array}{l}\text { The comb shape and the colors of } \\
\text { earlobe, feather and shank was studied. } \\
\text { The pea }(\mathrm{P}) \text {, rose }(\mathrm{R}) \text {, and crest }(\mathrm{Cr}) \\
\text { loci controlling the comb shape, the } \\
\text { barring (B), extension of black (E), } \\
\text { inhibitor (I) and silver (S) loci } \\
\text { controlling the feather color, and the Id } \\
\text { locus for shank color were } \\
\text { investigated. }\end{array}$ & $\begin{array}{l}\text { Production parameter-not } \\
\text { mention } \\
\text { The comb shape (the P, R and } \\
\text { Cr loci), earlobe color, feather } \\
\text { color (the B, E, I and S loci) } \\
\text { and the shank color (the Id } \\
\text { locus). Gene frequencies were } \\
\text { estimated at these } 8 \text { loci and } \\
\text { at four blood group loci (The } \\
\text { Ea-A, Ea-B, Ea-D and Ea-E loci). }\end{array}$ & Okada et al.,1987 \\
\hline
\end{tabular}




\begin{tabular}{|c|c|c|c|}
\hline $\begin{array}{l}\text { Location: } \\
\text { BLRI farm } \\
\text { Intensive } \\
\text { system }\end{array}$ & $\begin{array}{l}\text { Plumage color- black brownish } \\
(33.33 \%) \text { followed by white with black } \\
\text { tips }(28.33 \%) \text { and red brownish } \\
(18.33 \%) \text {. Shank color-whitish } 35 \% \\
\text { yellowish, } 31.38 \% \text {; black } 11.66 \% \text { and } \\
\text { others } 21.67 \% \text {. egg color- light brown } \\
(62.42 \%) \text { to cream or off white } \\
(30.28 \%)\end{array}$ & $\begin{array}{l}\text { Male: Shank Length }(\mathrm{cm})- \\
11.09 \text {, Wattle length }(\mathrm{cm})- \\
4.09 \text {, Body weight }(\mathrm{kg})-2.60 \\
\text { Number of eggs } / \text { hen from } \\
\text { starting to ten months of } \\
\text { laying }-104 \text {, egg weight } \\
40.32 \text { fertility }(\%) 91.55 \text {, } \\
\text { hatchability }(\%), 79.23\end{array}$ & Faruque et al., 2010 \\
\hline $\begin{array}{l}\text { Location: } \\
\text { BLRI farm } \\
\text { Intensive } \\
\text { system }\end{array}$ & Morphology- not mention & $\begin{array}{l}\text { Body weight at } 38 \text { weeks of } \\
\text { age }-1429 \mathrm{~g}, \quad \text { egg weight } \\
40.94 \mathrm{~g}, \quad \text { egg production } \\
27.23 \%\end{array}$ & Islam et al., 2004 \\
\hline $\begin{array}{l}\text { Location: } \\
\text { BLRI farm } \\
\text { Intensive } \\
\text { system }\end{array}$ & Morphology- not mention & $\begin{array}{l}\text { The live weight of the birds } \\
\text { fed PHPE diet was } \\
\text { significantly higher }(699 \pm 18) \\
\text { than that of MLPE diet } \\
(492 \pm 10) \text { at } 8 \text { weeks of age. } \\
\text { Feed conversion ratio (FCR) } \\
\text { was better in PHPE diet } \\
(2.89 \pm 0.03) \text { than in } \\
\text { MLPE diet }(3.22 \pm 0.09)\end{array}$ & Rahman et al., 2013 \\
\hline $\begin{array}{l}\text { Location: } \\
\text { Chittagong } \\
\text { Hill tract }\end{array}$ & Morphology-not mention & $\begin{array}{l}\text { EW-Hx, Fayoumi- } 42.35 \mathrm{~g} \\
\text { Egg shell thickness- } 0.35 \mathrm{~mm} \\
\text { Shape index- } 0.72\end{array}$ & Khan et al., 2004 \\
\hline $\begin{array}{l}\text { Semi- } \\
\text { scavenging } \\
\text { system }\end{array}$ & & & \\
\hline
\end{tabular}

EW - Egg Weight

\section{Population dynamics of three genotypes}

An In-depth survey was conducted in one hundred fifteen (115) households (HH) at Bandarban Sadar Upazila, Sarail Upazila and Baraigram Upazila where chickens were reared in situ. Information regarding the number of chickens per household, age and sex group (chick, pullet, cockerel, hen or cock), morphology of adult chickens (comb type, ear lobe color, shank color, plumage pattern), housing pattern, length of lighting practiced for laying hens, available feeds and feeding system, disease prevention measures and treatment practiced by the farmers were investigated.
The chickens were categorized in cock (male chicken $>8$ months), hen (female chicken $>8$ months), cockerels (male chick 2-8 months), pullet (female chick 2-8 months) and chicks (unsexed chick $<2$ months of age). The average number of chicken according to age and sex group is presented in Table 4. The average number of chicken per house-hold was almost same in Baraigram, Pabna and Sarail, Brahmanbaria (7.93 \pm 4.46 and $7.69 \pm$ 2.75); but was lower in Bandarban Hill district $(5.11 \pm 1.78)$. This variation was also observed in age and sex group of chicken in three localities as presented in Table 4. 
Table 4. Population dynamics of three genotypes

\begin{tabular}{l|ccc}
\hline \multirow{2}{*}{ Parameter } & \multicolumn{3}{c}{ Location } \\
\cline { 2 - 4 } Genotypes & Natore & Brahmanbaria & Bandarban \\
\cline { 2 - 4 } Chicken/house-hold & Indigenous & Aseel & Hilly \\
Cock/house-hold & $7.23+4.46$ & $7.69+2.75$ & $5.11+1.78$ \\
Hen/house-hold & $0.57 \pm 0.14$ & $1.69 \pm 0.31$ & $2.00 \pm 0.13$ \\
Cockerel /house-hold & $2.27 \pm 0.25$ & $3.31 \pm 0.27$ & $0.16 \pm 0.10$ \\
Pullet /house-hold & $1.33 \pm 0.19$ & 00.00 & $0.07 \pm 0.04$ \\
Chick/house-hold & $0.53 \pm 0.26$ & $0.08 \pm 0.04$ & $1.96 \pm 0.06$ \\
\hline
\end{tabular}

Morphological characteristics of Indigenous, Aseel and Hilly chicken as shown in Table 5.

Table 5: Phenotypic characteristics of Indigenous, Aseel and Hilly chicken

\begin{tabular}{|c|c|c|c|c|}
\hline \multicolumn{2}{|l|}{ Parameter } & \multicolumn{3}{|c|}{ Mean (\%) } \\
\hline \multicolumn{2}{|c|}{ Genotype } & Indigenous & Aseel & Hilly \\
\hline \multirow[t]{4}{*}{ Plumage color } & Black brownish & 35 & 30 & 50 \\
\hline & brown black strip & 30 & - & 45 \\
\hline & Completely black & 30 & 25 & 5 \\
\hline & Golden red & 5 & 45 & - \\
\hline \multirow[t]{3}{*}{ Shank color } & white & 90 & - & 39 \\
\hline & black & 10 & - & 11 \\
\hline & yellow & - & 100 & 50 \\
\hline \multirow[t]{2}{*}{ Comb color } & red & 99 & 100 & 100 \\
\hline & pale red & 1 & - & - \\
\hline \multirow[t]{4}{*}{ Comb type } & single & 99 & - & 88.9 \\
\hline & rose & - & 25 & - \\
\hline & pea & - & 75 & - \\
\hline & others & 1 & - & 11.1 \\
\hline \multirow[t]{2}{*}{ Ear lobe color } & red & 53.33 & 100 & 83.3 \\
\hline & white & 46.66 & - & 16.9 \\
\hline \multirow[t]{2}{*}{ Skin color } & white & 99 & 100 & 100 \\
\hline & yellow & 1 & - & - \\
\hline \multirow[t]{2}{*}{ Egg shell color } & white & 93.33 & 20 & 83.3 \\
\hline & light brown & 6.66 & 80 & 11.7 \\
\hline
\end{tabular}

Indigenous chicken have variation in plumage color. Black brownish $35 \%$, brown with black strip $30 \%$, completely black $30 \%$ and golden red 5\%. Aseel chickens have 45\% golden red, $30 \%$ black brownish, completely black $25 \%$ but Hilly chickens have $50 \%$ black brownish, $45 \%$ brown with black strip and $5 \%$ black plumage color. In case of shank color, 3 shank color were observed. The $90 \%$ of indigenous chicken had white shank and
$10 \%$ had black shank. All the Aseel chicken had yellow shank. In case of Hilly and Aseel chicken, $100 \%$ comb color was red color comb while $99 \%$ of Indigenous chicken had red color comb and the rest had pale red color comb. $99 \%$ of Indigenous chicken had single comb. $75 \%$ of Aseel had pea comb and $25 \%$ had rose comb. $88.9 \%$ of Hilly had single comb and $11.1 \%$ had buttercup comb. $53.33 \%$ Indigenous chicken had red color 
earlobe and the rest had white earlobe; $100 \%$ Aseel had red earlobe; for Hilly chicken, $83.3 \%$ had red earlobe and the rest had white earlobe. Aseel hens laid 80\% light brown and $20 \%$ white color eggs; Hilly hens laid $83.3 \%$ white and $11.7 \%$ light brown eggs. Indigenous hens laid mainly white colored eggs.

Hilly chickens are covered with plumage of white with black tips $85 \%$ followed by multicolor $15 \%$. According to Tabassum (2012) described indigenous chickens were multiple colored were $24 \%$ and white $\&$ red colored $1 \%$. The result of present study is more explanatory than the previous ones. In case of shank color 3 shank colored chickens were found in studied villages. The shank of indigenous chicken was $90 \%$ white and $10 \%$ black. While the shank color of Aseel chicken was observed 100\% yellow. The shank color of Hilly chicken was $50 \%$ yellow, $39 \%$ white and $11 \%$ was black. Tabassum (2012) described 4 shank colored; $52 \%$ white, $2 \%$ white $\&$ red, $36 \%$ black and $10 \%$ yellow in indigenous chickens. Daikwo et al. (2011) recorded $8.5 \%$ white, $13.75 \%$ black, $37.25 \%$ black/yellow and $40.5 \%$ yellow. Sarker et al. (2014), reported the most predominant shank color was white in forest ecotype but grey, black \& yellow colored shanks were also found and all the chickens had yellowish shank color in Aseel chicken in Bangladesh. The results are not consistent with the observations of others except Sarker et al. (2010). In case of Hilly and Aseel chicken $100 \%$ comb color was red but for Indigenous 99\% was red and rest was pale red color. The comb type of Indigenous was $99 \%$ single and $1 \%$ others; Aseel was
$75 \%$ pea and $25 \%$ rose comb; Hilly was $88.9 \%$ single and $11.1 \%$ others. The single comb was the commonest (96.45\%), followed by rose $(3.10 \%)$ while pea was the least $(0.44 \%)$ reported by Apuno et al. (2011). Badubi et al. (2006) reported that the Indigenous chickens were mostly single combed as was also observed by (Bhuiyan et al., 2005) in Asia among the Indigenous chickens of Bangladesh. Thus the results of present study and published reports from others research works suggested that the single comb is dominant over any type of combs elsewhere. The earlobe color of Indigenous was $53.33 \%$ red and $46.66 \%$ was white; for Aseel $100 \%$ was red and for Hilly $83.3 \%$ was red and $16.9 \%$ was white which are similar to the findings of Biswas (2005) reported that the red earlobe color of Indigenous chicken was predominantly red $(58 \%)$ followed by white earlobe $(45.8 \%)$ but Ahmed and Ali (2007) however found $80.55 \%$ white earlobe color of indigenous chicken. In case of skin color, the result shows that $99 \%$ was white in Indigenous chicken and $1 \%$ was yellow but in case of Hilly and Aseel $100 \%$ was white. Tabassum (2012) described white (89.9\%) skin colored was prominent and yellow skin colored chicken also available. The result shows that Aseel chickens laid $80 \%$ light brown \& 20\% white color eggs; Hilly chickens $83.3 \%$ white \& $11.7 \%$ light brown eggs. Indigenous chicken mainly laid white $(93.33 \%)$ colored eggs and light brown (6.66\%); which is similar findings of Tabassum (2012). Biswas (2005) reported that the indigenous chickens laid light brown (62.42\%) to cream of off white $(30.28 \%)$ colored eggs. 
Table 6: Management practices for Indigenous, Aseel and Hilly chicken

\begin{tabular}{|c|c|c|c|c|}
\hline \multirow[t]{2}{*}{ Parameter } & & \multicolumn{3}{|c|}{ Genotype } \\
\hline & & Indigenous & Aseel & Hilly \\
\hline \multicolumn{2}{|c|}{ Management system on the basis of input supply } & $\begin{array}{l}\text { Semi- } \\
\text { intensive }\end{array}$ & $\begin{array}{l}\text { Semi- } \\
\text { intensive } \\
*\end{array}$ & $\begin{array}{l}\text { Semi- } \\
\text { intensive }\end{array}$ \\
\hline \multirow{3}{*}{$\begin{array}{l}\text { Feeding } \\
\text { system }\end{array}$} & Only scavenging (\%) & 60 & 60 & 80 \\
\hline & $\begin{array}{l}\text { Scavenging }+ \text { one time supplement } \\
(\%)\end{array}$ & 35 & 35 & 20 \\
\hline & $\begin{array}{l}\text { Scavenging } \\
\text { supplement }(\%)\end{array}+$ two times & 5 & 5 & - \\
\hline \multirow{4}{*}{$\begin{array}{l}\text { Feed used as } \\
\text { supplement }\end{array}$} & Cooked rice $(\%)$ & 50 & 20 & 60 \\
\hline & Rice granule (\%) & 40 & 80 & 40 \\
\hline & Paddy (\%) & & 10 & - \\
\hline & Rice bran & 10 & - & - \\
\hline \multirow[t]{2}{*}{ Lighting system } & Artificial lighting (\%) & - & - & - \\
\hline & Natural lighting (\%) & 100 & 100 & 100 \\
\hline \multirow[t]{5}{*}{ House } & Readymade small house (\%) & 30 & 30 & - \\
\hline & Homemade earthen house (\%) & 30 & - & - \\
\hline & Wooden house $(\%)$ & 10 & 35 & - \\
\hline & Bamboo house $(\%)$ & - & 25 & 40 \\
\hline & $\begin{array}{l}\text { Kept in case in night time in bed } \\
\text { room/store room } / \text { kitchen }(\%)\end{array}$ & 30 & 40 & 60 \\
\hline \multirow{4}{*}{$\begin{array}{l}\text { Housing } \\
\text { materials }\end{array}$} & Mud & 40 & - & - \\
\hline & Wood & 20 & 40 & 10 \\
\hline & Tin & 20 & & \\
\hline & Bamboo & - & 60 & 90 \\
\hline \multirow[t]{8}{*}{ Treatment } & Regular vaccination $(\%)$ & 26.34 & 26.34 & - \\
\hline & Partial vaccination $(\%)$ & 20.00 & 20.00 & - \\
\hline & No vaccination $(\%)$ & 53.66 & 53.66 & 100 \\
\hline & Regular de-worming (\%) & 26.34 & 26.34 & - \\
\hline & Partial de-worming (\%) & 20.00 & 20.00 & \\
\hline & No de-worming $(\%)$ & 53.66 & 53.66 & \\
\hline & $\begin{array}{l}\text { Treatment done by Veterinary } \\
\text { expert }(\%)\end{array}$ & 20 & 20 & - \\
\hline & Non- veterinary expert (\%) & 80 & 80 & - \\
\hline
\end{tabular}

*Breeding male and fighter males were kept in confined for 24 hours and fed in confinement

\section{Management system}

Majority of indigenous chickens in Bangladesh are reared in the scavenging production systems. Different authors reported that the most common system was scavenging type being characterized as small flock sizes, beside this semi-intensive production system also used. Most of the caretaking practices of local chicken husbandry and being undertaken by women and children of household in Bangladesh. A shelter used by the majority of the farmers for indigenous chicken productions is sharing the house with the family at night. There is no planned feeding system for 
chickens and almost the only source of diets is scavenging feed resource. Moreover, there is no planned breeding also. Perpetuation of the indigenous chicken is by natural incubation process. A broody hen is engaged in hatching and rearing the chicks. Most of producers rear their indigenous chickens to generate incomes by selling eggs and marketable chickens.

Generally, chickens picked up grains such as rice, vegetables, green grass, insect, earthworm etc. from the yard, as chickens were reared under semi-intensive system. Farmers supplied feed 2 times daily and maximum supplied only a carbohydrate source: that is broken rice, wheat, rice polish etc. Chickens in the study area mostly depended on scavenging feed that were insufficient for their requirement and contained low nutrient. Huque et al. (1992) reported that native chickens consumed 9-27 $\mathrm{g} /$ bird/day scavenge able feedstuffs, which is lower than standard requirement and contained low nutrients, and may be one of the important factors that cause low productivity of local chickens (Das et al., 2008).

The survey and the findings of different writers revealed that management was semi-intensive system for all the chickens except breeding and fighting cocks of Aseel in Sarail. Breeding and fighting cocks of Aseel in Sarail were kept in confinement for 24 hours, and management system was intensive. Feeds were not usually supplemented in all three genotypes reared under scavenging system. Chickens picked up grains such as rice, vegetables, green grass, insect, earthworm etc. from the yard, as chickens were reared under scavenging system. Different types of housing were used for chickens in the selected sites. Approximately $30 \%, 40 \%$ and $60 \%$ of farmers kept chickens in their living houses in cases of Indigenous chickens, Aseel and Hilly chicken, respectively. No farmer used artificial lighting for the chickens. $53.66 \%$ of the farmers did not vaccinate their chickens, whereas the rest vaccinated their chickens once or twice per year. The vaccination programs were provided by mainly nonveterinary expert.

$100 \%$ management system was semi-intensive. Approximately $30 \%$ of farmers kept chickens in their living houses. 50\% houses is made in earthen, $37 \%$ in wooden houses and 13\% houses was made by tin for indigenous chickens. In case of Aseel 50\% houses was made by bamboo and $50 \%$ made by wooden. $100 \%$ of farmers did not use lighting and ventilation system. $73.33 \%$ of the farmers in selected areas did not vaccinate their chickens, whereas the remainder vaccinated once or twice per year. The vaccination programs are mainly provided by local livestock personnel and other experts. All of farmers were selling their chickens by indirect marketing systems.

\section{Conclusion}

The present findings reveal that all Indigenous chickens may have production potentialities. Aseel is significantly different from others indigenous chickens in all phenotypic parameters. It seems that the situation for indigenous poultry production is still remain problematic, so that some sorts of technological intervention are required. Rural poultry production should be improved by proper nutritional inputs. Conservation of indigenous germplasm by proper planning is important and development of a local breed or variety by using these indigenous 
chickens is necessary. Veterinary services should be also strengthened for the diagnosis of diseases.

\section{References}

Ahmed, F.M., Nishibori M. and Islam M.A. 2012. Production and price of indigenous naked neck and full feathered chicken reared under rural scavenging system in Bangladesh. Journal of Agricultural Extension and Rural Development Vol. 4: (4)-: 92-97.

Apuno, A.A., Mbap S.T. and Ibrahim T. 2011. Characterization of local chickens (Gallus gallusdomesticus) in Shelleng and Song Local Government areas of Adamawa State, Nigeria. Agriculture and Biology Journal of North America. 2 (1): 6-14.

Aziz, M.A., Miah, M.A.H. 2003. Family poultry farming system in developing countries, 3rd International Poultry Show \& Seminar.

Badubi, S.S., Rakereng, M., and Marumo, M. 2006. Morphological characteristics and feed resources available for Indigenous chickens in Botswana. Livestock Research for Rural Development 18 (1).

Bhuiyan, A.K.F.H., Bhuiyan, M.S.A. and Deb, G.K. 2005. Indigenous chicken genetic resources in Bangladesh: current status and future outlook. Animal Genetic Resources Information, FAO, Italy, 36: 73-84.

Billah, S.M. Nargis, F., Hossain, M.E., Howlider, M. AE.R. and Lee, S.H. 2013. Family poultry production and consumption pattern in selected households of Bangladesh. Journal of Agricultural Agri. Extension.and Rural development develop. 5: 62-69.

Biswas, S.R. 2005. Genetic dilution of indigenous chicken in selected villages. MS Thesis, Department of Poultry Science, Bangladesh Agricultural University, Mymensingh.

Daikwo, I.S., Okpe, A.A. 2011. Phenotypic characterization of local chickens in Dekina., International Journal of Poultry Poult .Science Sci. 10 (6): 444 - 447.
Das, S.C., Chowdhury, S.D., Khatun, M.A., Nishibory, M., Isobe, N. and, Yoshimura, Y. 2008. Poultry production profile and expected future projection in Bangladesh., World's Poultry Science Sci. Journal 64 (1): 99-118.

Dutta, R.K. Saiful, I.M. and Ashraful, K.M. 2013. Production Performance of Indigenous Chicken (Gallus domesticusL.) in Some Selected Areas of Rajshahi, Bangladesh. American Journal of .Experimental Exp. Agriculture Agri. 3(2): 308323.

Ershad, S.M.E. 2005. Performance of hybrid layers and native hens under farmers management in a selected area of Bangladesh. International Journal of Poultry Poult. Science Sci. 4(4): 228-232.

Faruque, M.O., Hasnath M.A., Khan M.Y.A. Takahashi, Y. and Nomura .Kamano. T. 2010. Current status of animal genetic resources and livestock production in Bangladesh., Report of the society for researches on native livestock, 25:1-33.

Faruque, S. Siddiquee N.U., Afroze, M.A. and Islam, M.S. 2010. Phenotypic characterization of native chicken reared under intensive management system. J. Bangladesh Agri.Univ. 8:79-82.

Faruque, S., Buiyan A.K.F.H., Rahman M.M. and, Islam M.N. 2013. Improvement of indigenous chickens of Bangladesh: Performance of 1st generation.8th International Poultry Show and Seminar, Bangabandhu International Conference, Center (BICC), Dhaka, Bangladesh, 28 Feb- 2 March

Hossain, M. M. and Sarwer, R. H. 2007. Improvement of livelihood of resource poor women through poultry rearing in two districts of Bangladesh. 5th International Poultry Show \& Seminar, 1-3 March.

Huque, M.I. Faruque, M.O., Chowdhury, S.D., Hoque, M. A. Majumder, M. K. H. and Hossain M. S. 2013. Distribution pattern and phenotypes of Aseel chicken in Brahmanbaria district. Proceedings of the 2nd National Seminar, 25th April, Bangladesh Agricultural Universitry, Mymensingh-2202.

Huque, Q. M. E., Ukil, M. A., Hossain, M. J., and Haque, M.E. 1992. Nutritional status of 
scavenging hens in Bangladesh. Bangladesh Journal of Scientific Research (10): 217-222.

Islam, M.N. Azmal S.A. and Uddin, M.S. 2004. Study on the quantitative and morphological characteristics of native chicken in Bangladesh. Annual Research Review Workshop, Bangladesh Livestock Research Institute, Savar, Dhaka-1341.

Islam, M.Z. 2011. Morphological classification of indigenous chicken and their relation with production potential.MS Thesis, Department of Poultry Science, Bangladesh Agricultural University, Mymensingh.

Khan, M.K.I., Khatun, M.J. and Kibria A.K.M.J, 2004. Study the quality of eggs of different genotypes of chickens under semi-scavenging system of Bangladesh, Pakistan Journal of Biological Biol. Science, .7(12): 2163-2166.

Nipa, R.S. Azharul, H., Shakila F., Nazrul, I. and Bhuiyan A.K.F.H. 2012. An ex situ study on body characteristics and effect of plumage color on body weight of indigenous chicken (Gallus domesticus) in Bangladesh. Department of Animal Breeding and Genetics Bangladesh Agricultural University, Mymensingh-2202.

Okada, I., Maeda, Y., Ota K., Takao, N., Amano, T., Yaetsu, K., Hasnath, M. A., Faruque, M.O., Majid, M.A. and Karim M.D.R. 1987. Genetically studies on native chickens in Bangladesh. Genetic studies on breed differentiation of the native domestic animals in Bangladesh, 2:11-26.

Paul, D.C., Huque, Q.M.E., Islam, M.R. and Jalil, M. A. 2003. Organic chicken farming- a tool for family nutrition and cash generation: Bangladesh perspective. 3rd International Poultry Show \& Seminar.

Rahman, M.M., Faruque, S., Islam, .M.S. Islam, M.N. and Rashid, M.A. 2013. Comparison of growth performance and meat yield of Hilly Chicken under two feeding regimens. The Agriculturists. 11: 38-43.

Sarker, K. 2007. A transition from subsistence to semi-comertial family poultry farming with indigenous chickens.Central Poultry Farm, Mirpur, DLS, 5th International Poultry Show \& Seminar, 1-3 March.
Sarker, K. 2013. Performance and profitability of feeding commercial diets to indigenous chicks. 8th international poultry show and seminar.

Sarker, N.R. Islam M.S., Uddin, M.S. and Huque Q. M.E. 2005. Production pattern of native chicken in some selected villages in north-western district of Bangladesh. 4th International Poultry Show \& Seminer, 10-12 March.

Sarker, N.R., Hoque, A., Faruque, S., Islam, M.N., Bhuiyan, A.K.F.H. 2014: An ex-situ study on body characteristics and effect of plumage color on body weight of indigenous chicken (Gallus domesticus) in Bangladesh. Acta Scientiarum. Animal Sciences 36 (1): 79-84.

Sarker, M.J.A. 2011.Phenotypic Characterization of Aseel Chicken of Bangladesh.M.S.thesis, Department of Animal Breeding and Genetics, Bangladesh Agricultural University, Mymensing2202, Bangladesh.

Shahjahan, M. 2010. Diversity in performance of indigenous chicken in some selected areas of Bangladesh in-situ.M.S. thesis, Department of Animal Breeding and Genetics, Bangladesh Agricultural University, Mymensing-2202, Bangladesh. 7th International Poultry Show \& Seminar, 25-27 March.

Tabassum, F. 2012. An in-depth study on morphometric and phenotypic characteristics of indigenous chicken. M.S. thesis, Department of Animal Breeding and Genetics, Bangladesh Agricultural University, Mymensing - 2202, Bangladesh.

Yamamoto, Y., Ali, A., Amin, M.R., Khan M.Y.A., Hussain S. S. Faruque, M. O. and Amano, 2010. Composition of the genes controlling blood groups and morpho-genetic traits of Bangladesh native chicken and its phylogenic study. Report of the society for researches on native livestock, 25: 131-146. 